\title{
The triple system KR Comae Berenices ${ }^{\star}$ (Research Note)
}

\author{
P. Zasche ${ }^{1}$ and R. Uhlár ${ }^{2}$ \\ 1 Astronomical Institute, Charles University, Prague, V Holešovičkách 2, 18000 Prague 8, Czech Republic \\ e-mail: zasche@sirrah.troja.mff.cuni.cz \\ 2 Private Observatory, Pohoří 71, 25401 Jílové u Prahy, Czech Republic \\ Received 29 April 2010 / Accepted 12 July 2010
}

\section{ABSTRACT}

\begin{abstract}
Aims. We present the detailed analysis of triple system KR Com with different observational techniques - photometry, interferometry, and period variation.

Methods. The use of $B V R$ photometry of the close-contact binary KR Com, which is the primary component of a triple system, helps us to better describe the properties of the components. The interferometric data obtained during the last 30 years sufficiently determine the visual orbit, but the use of minima timings of KR Com for the study of period variation together with the visual orbit is a novel approach in this system.

Results. Basic physical parameters resulting from the light curve analysis agree well with the previous results from spectroscopy. The temperatures for the primary and secondary component resulted in 5549 and $6072 \mathrm{~K}$, respectively, and the amount of the third light in all filters is about $1 / 3$ of the total luminosity. The distant third component revolves around the common barycenter on $11 \mathrm{yr}$ orbit with a very high eccentricity (0.934) and this movement is also detectable via the period variation, which is clearly visible in the $\mathrm{O}-\mathrm{C}$ diagram of times of minima observations. The use of minima times for the combined analysis helps us to independently determine the distance to the system $(64.02 \pm 9.42 \mathrm{pc})$ and also to confirm the orientation of the orbit in space.

Conclusions. New minima observations and also spectroscopy would be very profitable, especially during the next periastron passage in the year 2017.
\end{abstract}

Key words. binaries: eclipsing - binaries: visual - stars: fundamental parameters - stars: individual: KR Com

\section{Introduction}

Multiple stellar systems (i.e., of a multiplicity of three and higher) are excellent objects to be studied. Besides the statistics and their relative frequency among the stars, it is important to investigate these systems in detail, also because of their stellar evolution, their origin, to test the influence of the distant components to the close pair, etc.

One of these systems is KR Com (HD 115955, HIP 65069), which is also A component of the visual binary A 2166 (WDS J13202+1747). Its relative brightness is about $7.2 \mathrm{mag}$ in $V$ filter and its combined spectral type has been classified as F8V, Abt (1981). The system has been discovered as a photometrically variable one from the Hipparcos data by Selam (2004). Its orbital period is about 0.408 days only, but both eclipses are rather shallow (because of a close bright companion). Although both minima are of similar depths, the system has been incorrectly classified as a $\beta$ Lyrae one, Kazarovets et al. (1999).

The close binary system has been extensively studied spectroscopically by Rucinski et al. (2002). The radial velocity curve of KR Com has been derived and also an influence of the third body in the system to the close pair and its properties has been discussed in subsequent papers by e.g., D'Angelo et al. (2006). A very low value of the mass ratio of this system has been found, about 0.09 only, which makes KR Com an exceptional

* Photometric tables are only available in electronic form at the CDS via anonymous ftp to cdsarc.u-strasbg.fr (130.79.128.5) or via

http://cdsweb.u-strasbg.fr/cgi-bin/qcat?J/A+A/519/A78 case among the $\mathrm{W}$ UMa-type binaries. The photometry and light curve solution of the close pair is still missing, which led us to observe this interesting target.

On the other hand, the close visual companion B (with an angular distance about only $0.1^{\prime \prime}$ ) was detected in 1980 by McAlister et al. (1983). The movement around a barycenter is very fast, its orbital period is about $11 \mathrm{yr}$ and the eccentricity is very high about 0.9 . The orbit has been derived by Hartkopf et al. (1996). Owing to the small angular separation between both components, any photometric and also spectroscopic observations cannot be done only for KR Com, and the influence of the third component has to be considered in the analysis. The eclipsing binary is the brighter component ( $\Delta m \sim 0.6 \mathrm{mag}$ ), and Rucinski et al. (2002) give the luminosity ratio $L_{3} /\left(L_{1}+L_{2}\right)=$ 0.56 . They also presented a broadening function, which indicated a slowly rotating third component, and in D'Angelo et al. (2006) the authors provided an estimate about the third-body mass $M_{3}=1.19 M_{\odot}$, while the individual masses of the close binary are 1.42 and $0.129 M_{\odot}$.

Thanks to the unusually low mass ratio of the eclipsing pair and the presence of the close interferometric component $\mathrm{B}$ we deal with a unique system, which is very useful to be studied in detail.

\section{Photometry}

Owing to its relatively high brightness, the system has not been observed photometrically and studied in detail. There exist the light curves from the Hipparcos data $\left(H_{p}\right.$ filter), and 


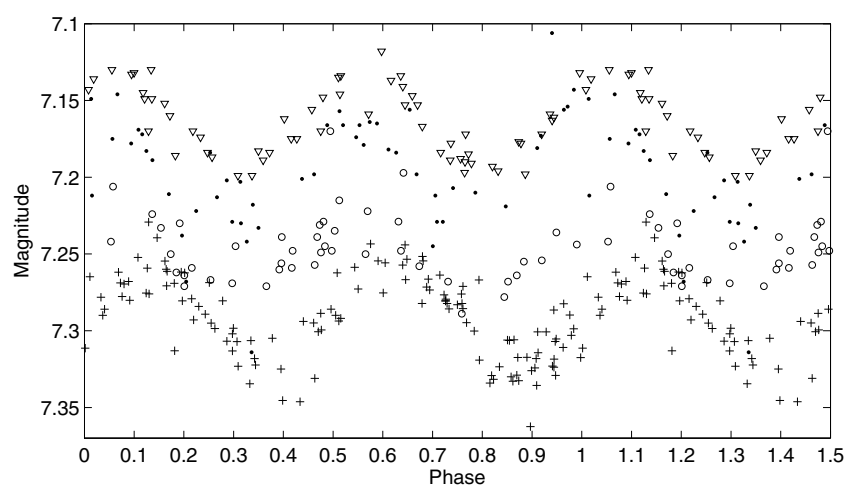

Fig. 1. Observations of KR Com from Hipparcos (plus) and ASAS (circles from 2009, dots from 2005, and triangles from 2003).

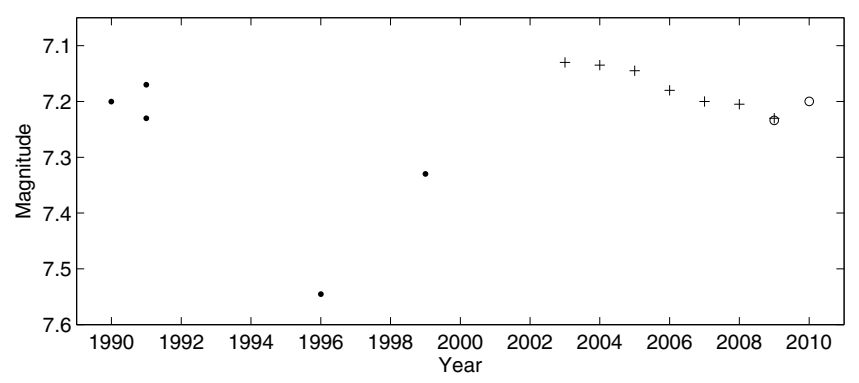

Fig. 2. Long-term variation of brightness of KR Com (in quadratures). Dots represent the various catalog data, the plus signs stand for the ASAS data, and the open circles mark our observations.

from the ASAS survey ( $V$ filter), see Fig. 1. Because of the low photometric amplitude and high scatter, these can hardly be used for any analysis of the light curve.

Thanks to the observations obtained by ASAS, which cover more than six years, we have found that there is a long-term photometric decrease of KR Com. It can be seen in Fig. 1, where we present the light curves from from different epochs. As one can see, a steady decrease of its brightness is on the order of 0.1 mag during the last six years.

The nature of this long-term behavior is questionable, as is which component of the system yields this variation. On the other hand, we have tried to find some historical data in various catalogs, which contain at least some information about its magnitude in photometric filters. VIZIER ${ }^{1}$ lists 145 catalogs containing several data about KR Com, from which a few usable data points were selected. The most complete are the measurements in the $V$ filter. In Fig. 2 we have plotted the data after 1990. The long-term variation is clearly visible, nevertheless this plot has a few flaws. For some of the points the photometric filter was not exactly the Johnson's $V$ filer, but rather some "similar" one. And secondly, for some of the data points it was quite hard to find an exact date of the observation, only a mean epoch is presented. The most deviating point from 1996 is a typical one. This particular data point comes from "The PM2000 Bordeaux proper motion catalogue" (Ducourant et al. 2006), and in its description is mentioned that the filter used is a non-standard one, a combination of two different filters. Exactly the same problem arises for the second deviating point from 1999, which comes from the "M2000: An astrometric catalog in the Bordeaux Carte du Ciel" (Rapaport et al. 2001). However, both these magnitudes use the same combination of filters and the difference between them during three years is easily visible. A period of this variation could

${ }^{1}$ http://vizier.u-strasbg.fr/viz-bin/VizieR only be vaguely estimated, but it is probably longer than the period of the third body.

One can speculate about the magnetic activity cycles in the system. Because of the spectral type (F8V), these can play a role, see Hall (1989). These cycles could slightly modulate the orbital period of the close pair. Moreover, accepting this hypothesis, according to Applegate (1992) there is also a modulation of the luminosity of the star on the order of $\delta L / L \sim 0.1$. If we propose that the most luminous component (the primary one) undergoes this variation, a variability of the brightness of the whole system results in only about $0.05 \mathrm{mag}$, which is much lower than observed since 1990. On the other hand, removing these two questionable data points discussed above, one can obtain a variation with an amplitude of about only $0.1 \mathrm{mag}$, which could be described by this mechanism. Therefore, the nature of this variation still remains an open question.

We have observed the system during the seasons 2009 and 2010. In total there are 17 nights of observations, but for the light-curve analysis we used only 7 nights of observations obtained from March 2010 to April 2010 and carried out with the same telescope and detector at the private observatory by one of the authors (RU). Owing to high brightness of the target, the refractor with a diameter of only $75 \mathrm{~mm}$ was used, equipped with the G2/KAF 0402ME CCD camera and standard $B, V$, and $R$ filters according to specification by Bessell (1990). All the measurements were processed by the software C-MUNIPACK ${ }^{2}$, which is based on aperture photometry and using the standard DaoPhot routines (Tody 1993).

The observations were transformed into the standard system using the well-known transformation equations ${ }^{3}$. Star HD 115981 was used as a comparison, while the check star to control the non-variability of the two was star HD 116206. The atmospheric extinction has been neglected because the stars are very close to each other (11 arcmin), their spectral types are also similar, and the observations have never been obtained below 25 deg above horizon. We used this approach because our observations have an accuracy not better than $0.01 \mathrm{mag}$ (for the scatter in individual filters see below), therefore any additional correction on the order of $0.001 \mathrm{mag}$ is practically useless for the transformation. The individual exposure times range from 25 to $140 \mathrm{~s}$.

The rest of observations were used only to determine the minima times for a period analysis. These new ones as well as the already published ones are given in Table 1, where the type of minima refers to the following ephemeris: $2454924.538+$ $0.4079711 \cdot E$. For all these observations, the Kwee-van Woerden method (hereafter KW, Kwee \& van Woerden 1956) was used for determining the time of minimum. This method is suitable for symmetric minima (which is the case for KR Com), because its principle is based on comparing the ascending and descending branch of the minima. We do believe that our error estimates are much more reliable than those already published because first, we did a detailed analysis of the KW result for each minimum, which means that we used different data sets (neglecting some of the observed points) and compared these results. And secondly, we also used a polynomial fitting to determine the time of minimum and compared this result with the KW one. The error of the particular minimum light was computed as a maximum difference between all the different results from different methods from the mean value. All minima times given in Table 1 are

\footnotetext{
2 See http://c-munipack. sourceforge.net/

3 See http://brucegary.net/AllSky/x.htm
} 
Table 1. Minima times of KR Com.

\begin{tabular}{|c|c|c|c|c|}
\hline HJD-2 400000 & Error & Type & Filter & Reference $^{a}$ \\
\hline 48500.2095 & 0.0075 & Pri & $\mathrm{Hp}$ & HIP \\
\hline 48500.4200 & 0.0129 & $\mathrm{Sec}$ & $\mathrm{Hp}$ & HIP \\
\hline 52638.8769 & 0.0165 & $\mathrm{Sec}$ & V & ASAS - [1] \\
\hline 52758.41911 & 0.00232 & $\mathrm{Sec}$ & V & ASAS \\
\hline 52758.62161 & 0.00301 & Pri & V & ASAS \\
\hline 53058.4796 & 0.0002 & Pri & $C$ & Krajci (2005) \\
\hline 53102.11786 & 0.00255 & Pri & V & ASAS \\
\hline 53150.2695 & 0.0005 & Pri & $C$ & Krajci (2005) \\
\hline 53475.0061 & 0.0004 & Pri & V & Nagai (2006) \\
\hline 53487.86332 & 0.00518 & $\mathrm{Sec}$ & V & ASAS \\
\hline 53488.05344 & 0.00767 & Pri & V & ASAS \\
\hline 53829.11608 & 0.00468 & $\mathrm{Sec}$ & V & ASAS \\
\hline 54225.87197 & 0.00849 & $\mathrm{Sec}$ & V & ASAS \\
\hline 54226.07038 & 0.00326 & $\mathrm{Sec}$ & $V$ & ASAS \\
\hline 54233.4095 & 0.0009 & Pri & $C$ & [2] \\
\hline 54580.40196 & 0.00273 & $\mathrm{Sec}$ & $V$ & ASAS \\
\hline 54613.0406 & 0.0002 & $\mathrm{Sec}$ & Ic & Nagai (2009) \\
\hline 54921.47502 & 0.00150 & $\mathrm{Sec}$ & $B$ & this paper \\
\hline 54921.47435 & 0.00246 & $\mathrm{Sec}$ & V & this paper \\
\hline 54921.47702 & 0.00178 & $\mathrm{Sec}$ & $R$ & this paper \\
\hline 54921.47534 & 0.00051 & $\mathrm{Sec}$ & $I$ & this paper \\
\hline 54924.53515 & 0.00043 & Pri & $B$ & this paper \\
\hline 54924.53553 & 0.00048 & Pri & V & this paper \\
\hline 54924.53559 & 0.00227 & Pri & $R$ & this paper \\
\hline 54924.53549 & 0.00031 & Pri & $I$ & this paper \\
\hline 54940.44566 & 0.00113 & Pri & $R$ & this paper \\
\hline 54971.44977 & 0.00098 & Pri & $B$ & this paper \\
\hline 54971.45562 & 0.00085 & Pri & $V$ & this paper \\
\hline 54971.45419 & 0.00052 & Pri & $R$ & this paper \\
\hline 54944.52261 & 0.00457 & Pri & $V$ & ASAS \\
\hline 54944.32228 & 0.00565 & $\mathrm{Sec}$ & V & ASAS \\
\hline 55259.67912 & 0.00319 & $\mathrm{Sec}$ & $R$ & this paper \\
\hline 55274.37115 & 0.00175 & $\mathrm{Sec}$ & $B$ & this paper \\
\hline 55274.36049 & 0.00135 & $\mathrm{Sec}$ & $I$ & this paper \\
\hline 55274.36870 & 0.00543 & $\mathrm{Sec}$ & $R$ & this paper \\
\hline 55274.35921 & 0.00329 & $\mathrm{Sec}$ & $V$ & this paper \\
\hline 55274.56764 & 0.00335 & Pri & $B$ & this paper \\
\hline 55274.56959 & 0.00143 & Pri & $I$ & this paper \\
\hline 55274.56684 & 0.00293 & Pri & $R$ & this paper \\
\hline 55274.56834 & 0.00264 & Pri & V & this paper \\
\hline 55280.49301 & 0.00093 & $\mathrm{Sec}$ & $B$ & this paper \\
\hline 55280.49220 & 0.00069 & $\mathrm{Sec}$ & $R$ & this paper \\
\hline 55280.48942 & 0.00109 & $\mathrm{Sec}$ & $V$ & this paper \\
\hline 55281.50792 & 0.00089 & Pri & $B$ & this paper \\
\hline 55281.50389 & 0.00085 & Pri & $R$ & this paper \\
\hline 55281.50817 & 0.00050 & Pri & V & this paper \\
\hline 55293.53729 & 0.00061 & Sec & $B$ & this paper \\
\hline 55293.53830 & 0.00157 & $\mathrm{Sec}$ & $R$ & this paper \\
\hline 55293.53559 & 0.00099 & $\mathrm{Sec}$ & $V$ & this paper \\
\hline 55294.56261 & 0.00110 & Pri & $B$ & this paper \\
\hline 55294.56418 & 0.00157 & Pri & $R$ & this paper \\
\hline 55294.56473 & 0.00120 & Pri & V & this paper \\
\hline 55357.38912 & 0.00235 & Pri & $I$ & this paper \\
\hline 55358.41029 & 0.00257 & $\mathrm{Sec}$ & I & this paper \\
\hline
\end{tabular}

Notes. ${ }^{(a)}$ HIP - Hipparcos observations, ASAS - data from the ASAS survey, [1] - see http://var . astro.cz/ocgate, [2] - unpublished yet, see http://eclipsingbinary.web.fc2 .com/vsnetmin.htm

heliocentric ones. Eleven new minima times were also derived from the data of the ASAS survey (Pojmanski 2002).

\section{The light curve analysis}

For the light-curve analysis we used the program PHOEBE (Prša \& Zwitter 2005), which is based on the Wilson-Devinney
Table 2. Light curve parameters of KR Com.

\begin{tabular}{cclc}
\hline \hline Parameter & Value & Parameter & Value \\
\hline$T_{1}[\mathrm{~K}]$ & $5549 \pm 244$ & $L_{1}(\mathrm{~B})[\%]$ & $50.67 \pm 0.20$ \\
$T_{2}[\mathrm{~K}]$ & $6072 \pm 270$ & $L_{2}(\mathrm{~B})[\%]$ & $17.75 \pm 0.19$ \\
$q\left(=M_{2} / M_{1}\right)$ & 0.091 (fixed) & $L_{3}(\mathrm{~B})[\%]$ & $31.58 \pm 0.20$ \\
$e$ & 0 (fixed) & $L_{1}(\mathrm{~V})[\%]$ & $47.63 \pm 0.24$ \\
$i[\mathrm{deg}]$ & $52.14 \pm 0.46$ & $L_{2}(\mathrm{~V})[\%]$ & $18.98 \pm 0.23$ \\
$x_{1}(\mathrm{~B})$ & 0.774 & $L_{3}(\mathrm{~V})[\%]$ & $33.39 \pm 0.24$ \\
$x_{2}(\mathrm{~B})$ & 0.699 & $L_{1}(\mathrm{R})[\%]$ & $47.05 \pm 0.72$ \\
$x_{1}(\mathrm{~V})$ & 0.635 & $L_{2}(\mathrm{R})[\%]$ & $19.04 \pm 0.67$ \\
$x_{2}(\mathrm{~V})$ & 0.565 & $L_{3}(\mathrm{R})[\%]$ & $33.90 \pm 0.72$ \\
$x_{1}(\mathrm{R})$ & 0.548 & \multicolumn{2}{c}{ Derived quantities: } \\
$x_{2}(\mathrm{R})$ & 0.485 & $R_{1}\left[\mathrm{R}_{\odot}\right]$ & $1.33 \pm 0.04$ \\
$g_{1}$ & $0.323 \pm 0.014$ & $R_{2}\left[\mathrm{R}_{\odot}\right]$ & $0.49 \pm 0.02$ \\
$g_{2}$ & $0.351 \pm 0.021$ & $M_{\text {bol, }, 1}[\mathrm{mag}]$ & $4.34 \pm 0.18$ \\
$A_{1}$ & $0.499 \pm 0.027$ & $M_{\text {bol, }, 2}[\mathrm{mag}]$ & $6.13 \pm 0.24$ \\
$A_{2}$ & $0.484 \pm 0.015$ & $\sigma[\mathrm{mag}](\mathrm{B})$ & 0.00745 \\
$F_{1}$ & $1.690 \pm 0.066$ & $\sigma[\mathrm{mag}](\mathrm{V})$ & 0.01583 \\
$F_{2}$ & $1.647 \pm 0.148$ & $\sigma[\mathrm{mag}](\mathrm{R})$ & 0.06737 \\
\hline
\end{tabular}

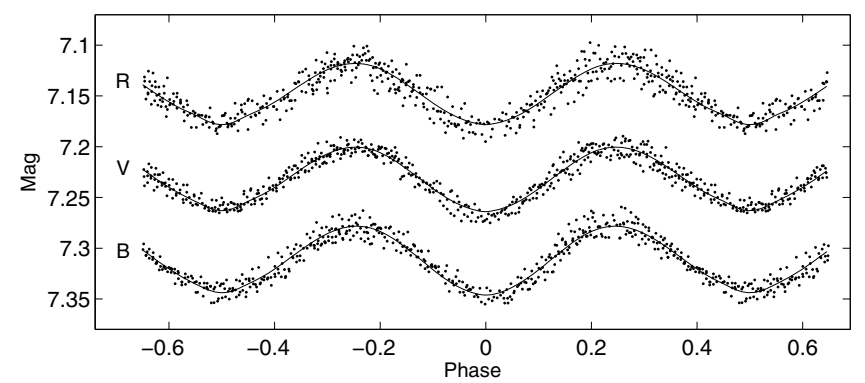

Fig. 3. Our new observations of the light curves of KR Com in three filters. The $B$ and $R$ curves are shifted for better clarity. The theoretical fits and parameters are described in the text and Table 2.

algorithm (Wilson \& Devinney 1971). The model atmospheres made by Kurucz (1996) were used. The derived quantities are: the individual temperatures $T_{1}$ and $T_{2}$, the inclination $i$, the luminosities $L_{i}$, the gravity darkening coefficients $g_{i}$, the limb darkening coefficients $x_{i}$, the albedo coefficients $A_{i}$, and the synchronicity parameters $F_{i}$. The limb darkening was approximated via linear cosine law, and the values of $x_{1}$ and $x_{2}$ were interpolated from the van Hamme's tables, van Hamme (1993).

Because the system has been analyzed spectroscopically in detail by Rucinski et al. (2002), we used the mass ratio value $q=M_{2} / M_{1}=0.091$ derived from spectroscopy for the analysis of our $B, V$, and $R$ photometry. The other relevant parameters of the light curve were computed (with fixed circular orbit) and the results are presented in Table 2 . The final fits and the photometric data are plotted in Fig. 3. The scatter in the $B$ and $V$ filters is about $0.010-0.015 \mathrm{mag}$, in the $R$ about $0.025 \mathrm{mag}$, and the numbers of data points are 451,468 , and 474 , for $B, V$, and $R$, respectively. The individual observational errors of the measurements were about 5-10 times lower than a typical peak-to-peak scatter of the measurements in the particular filter. The same applies for the measurement errors for the comparison and check stars. Their respective errors are also on the order of $0.003 \mathrm{mag}$ only, but these values represent merely the mathematical results of errors from the photometry of the stars. More plausible physical errors are much above these values, about $0.01 \mathrm{mag}$. This is actually a very similar situation as for the minimum estimate and the error of the KW method as discussed in the previous section. No additional short-time variation has been found in the 
data. The $B V R$ photometry used for the analysis is available in electronic form at the CDS.

The errors of the individual parameters were derived from the covariance matrix, following a standard procedure of error estimates, see e.g., "Numerical recipes. The art of scientific computing", Press et al. (1986).

From the light-curve analysis we see that the two components are in contact (A-type contact system), but their individual temperatures differ about more than $500 \mathrm{~K}$. On the other hand, the difference between the temperatures of $523 \pm 364 \mathrm{~K}$ is only marginally significant. We tried to find an appropriate solution with equal temperatures (which resulted in a value of about $6100 \mathrm{~K}$ ), but this solution resulted in $\chi^{2}$ about $13 \%$ worse than the solution presented in Table 2. Another approach was to fix the temperature of the primary to $T_{1}=6100 \mathrm{~K}$ and to fit only the secondary temperature. This resulted in fit with $\chi^{2}$ about $9 \%$ worse than our final solution. Both alternatives have been tested via an F-test to gauge how liable they are to introduce another free parameter (temperature) to the model. We concluded that introducing this new parameter is significant at a level about $86 \%$ and $84.5 \%$ for these two different approaches.

Our resulting values of parameters from the light curve fit can be compared with the estimates published in Rucinski et al. (2002). A value of the third light (33\% of total light) agrees well with their estimate (about $56 \%$ of $L_{1}+L_{2}$ ). The individual components are probably of $\mathrm{F}+\mathrm{G}$ spectral types for the primary and secondary, respectively. The secondary component has apparently undergone a mass transfer to the primary.

We were able to partly reveal the nature of the third body in the system via its temperature. This can be estimated thanks to the individual luminosities in the filters, from which one can derive the magnitudes in the particular filters. These values resulted in $B=8.96 \mathrm{mag}, V=8.39 \mathrm{mag}$, and $R=8.05 \mathrm{mag}$. As an independent comparison one can also check the value from the Washington Double Star Catalog (WDS) ${ }^{4}$, where the $V$ magnitudes 7.78 and 8.38 mag are given for the primary and secondary component of the visual pair, which excellently agrees with our value of $8.39 \mathrm{mag}$. From our estimates of the $B V R$ magnitudes, the photometric indices $B-V=0.57 \mathrm{mag}$ and $V-R=0.34 \mathrm{mag}$ can be compared with the color-temperature relations, e.g., by Houdashelt et al. (2000). This gives temperature estimate of the third component of $5900 \pm 200 \mathrm{~K}$, which indicates a spectral type between F8 and G6. On the other hand, if we try to derive also the radius of this component from the relation between the radii, luminosities and temperatures $R_{3}=\sqrt{L_{3} / L_{1} \cdot\left(T_{1} / T_{3}\right)^{4}} \cdot R_{1}$, we obtain values from 0.93 to $0.99 R_{\odot}$, which indicates a later spectral type, about G8-K1 (Harmanec 1988).

\section{Combined solution for the visual orbit and period variations}

As we mentioned above, the A-B system revolves around a barycenter with a period about $11 \mathrm{yr}$. Because this orbit has been derived about 15 years ago and many new observations are available since then, there is a need for new up-to-date solution for this orbit.

We used all available interferometric observations for the analysis of the pair collected in the "Fourth Catalog of Interferometric Measurements of Binary Stars ${ }^{5}$ ", Hartkopf et al. (2001). There are 25 usable measurements (see Table 3). In some

\footnotetext{
4 http://ad.usno.navy.mil/wds/

5 http://ad.usno.navy.mil/wds/int4.html
}

Table 3. Interferometric observations of A-B pair.

\begin{tabular}{cccc}
\hline \hline Date & $\begin{array}{c}\theta \\
{[\mathrm{deg}]}\end{array}$ & $\begin{array}{c}\rho \\
\text { [arcsec] }\end{array}$ & References \\
\hline 1980.1566 & 0.9 & 0.145 & McAlister et al. (1983) \\
1980.4817 & 2.0 & 0.142 & McAlister et al. (1983) \\
1983.0510 & 10.0 & 0.117 & McAlister et al. (1987) \\
1983.0701 & 12.2 & 0.106 & McAlister et al. (1987) \\
1984.0558 & 22.6 & 0.059 & Hartkopf et al. (1996) \\
1986.4067 & 327.7 & 0.058 & McAlister et al. (1989) \\
1987.1194 & 345.5 & 0.076 & Fu et al. (1997) \\
1987.2642 & 340.2 & 0.083 & McAlister et al. (1989) \\
1987.2859 & 343.0 & 0.089 & Fu et al. (1997) \\
1987.2886 & 348.8 & 0.089 & Fu et al. (1997) \\
1987.2914 & 346.8 & 0.089 & Fu et al. (1997) \\
1988.1022 & 345.5 & 0.097 & Fu et al. (1997) \\
1989.2300 & 353.6 & 0.117 & McAlister et al. (1990) \\
1990.2081 & 357.9 & 0.132 & Balega et al. (1994) \\
1990.2621 & 356.0 & 0.136 & Hartkopf et al. (1992) \\
1990.2759 & 356.0 & 0.135 & Hartkopf et al. (1992) \\
1991.25 & 357.0 & 0.136 & Perryman \& ESA (1997) \\
1991.3187 & 0.7 & 0.140 & Hartkopf et al. (1994) \\
1992.3098 & 4.3 & 0.138 & Hartkopf et al. (1994) \\
1992.3127 & 4.2 & 0.139 & Hartkopf et al. (1994) \\
1993.1971 & 7.7 & 0.128 & Hartkopf et al. (1994) \\
2002.3224 & 359.8 & 0.140 & Horch et al. (2008) \\
2002.3224 & 0.0 & 0.140 & Horch et al. (2008) \\
2002.3224 & 0.0 & 0.139 & Horch et al. (2008) \\
2006.1917 & 17.8 & 0.077 & Mason et al. (2009) \\
\hline & & &
\end{tabular}

of these observations the position angle $\theta$ has to be changed by $180^{\circ}$ (i.e., quadrant change, interchange of the components), the values presented in Table 3 are the corrected ones.

A movement of the contact binary around the barycenter with the distant component has to produce a periodic variation of orbital period of KR Com, a well-known "light-time effect" (hereafter LITE), see Irwin (1959). The amplitude of the LITE depends on the inclination of the $11 \mathrm{yr}$ orbit, the individual masses and also on the distance of KR Com. The distance from Hipparcos was originally derived as $76.51 \pm 5.46 \mathrm{pc}$, i.e., parallax $\pi=13.07 \pm 0.87$ mas (Perryman \& ESA 1997), but the more recent value is $83.97 \pm 5.32 \mathrm{pc}$, i.e., parallax $\pi=11.91 \pm$ 0.71 mas, van Leeuwen (2007). Nevertheless, the value of the parallax from the Hipparcos satellite could also be influenced by the movement on the long orbit. During the run of the satellite, the change of the position angle of the distant component was only about $15^{\circ}$, because in that time the B component was near apastron on its orbit. On the other hand, if one compares the error of its parallax with the other stars from the Hipparcos catalog with the similar parallaxes, the error is apparently not deviating significantly.

For an analysis of the period changes of KR Com we collected all available minima observations. The very first ones are from Hipparcos data (recalculated primary minimum and derived also a secondary one), while the most recent ones are our new observations of 2010. All these heliocentric minima times are given in Table 1 . We found that all the published minima times have their respective errors strongly underestimated (10 times or even more).

We used a similar approach as described in Zasche \& Wolf (2007) for analyzing the interferometric data together with the times of minima. The only difference was a calculation of both amplitudes - the semimajor axis of the visual orbit and the semiamplitude of LITE in the $\mathrm{O}-\mathrm{C}$ diagram. The main advantage of this approach is that we can independently calculate the distance 
Table 4. Final parameters of the long orbit.

\begin{tabular}{cc}
\hline \hline Parameter & Value \\
\hline$H J D_{0}$ & $2453058.4640 \pm 0.0021$ \\
$P$ [day] & $0.40797003 \pm 0.00000239$ \\
$p_{3}$ [day] & $4011.0 \pm 98.4$ \\
$p_{3}[\mathrm{yr}]$ & $10.98 \pm 0.27$ \\
$T_{0}$ & $2442055.1 \pm 118.0$ \\
$a$ [arcsec] & $0.1131 \pm 0.0189$ \\
$\omega_{3}[\mathrm{deg}]$ & $301.8 \pm 3.8$ \\
$e_{3}$ & $0.9340 \pm 0.0239$ \\
$i[\mathrm{deg}]$ & $67.68 \pm 2.03$ \\
$\Omega$ [deg] & $210.03 \pm 1.25$ \\
$A[\mathrm{day}]$ & $0.0171 \pm 0.0019$ \\
\hline$f\left(M_{3}\right)\left[M_{\odot}\right]$ & $0.3262 \pm 0.0896$ \\
$M_{3}\left[M_{\odot}\right]$ & $1.598 \pm 0.423$ \\
$a_{12}[\mathrm{AU}]$ & $3.677 \pm 0.821$ \\
$a_{3}[\mathrm{AU}]$ & $3.563 \pm 1.027$ \\
$\pi[\mathrm{mas}]$ & $15.62 \pm 1.80$ \\
\hline
\end{tabular}

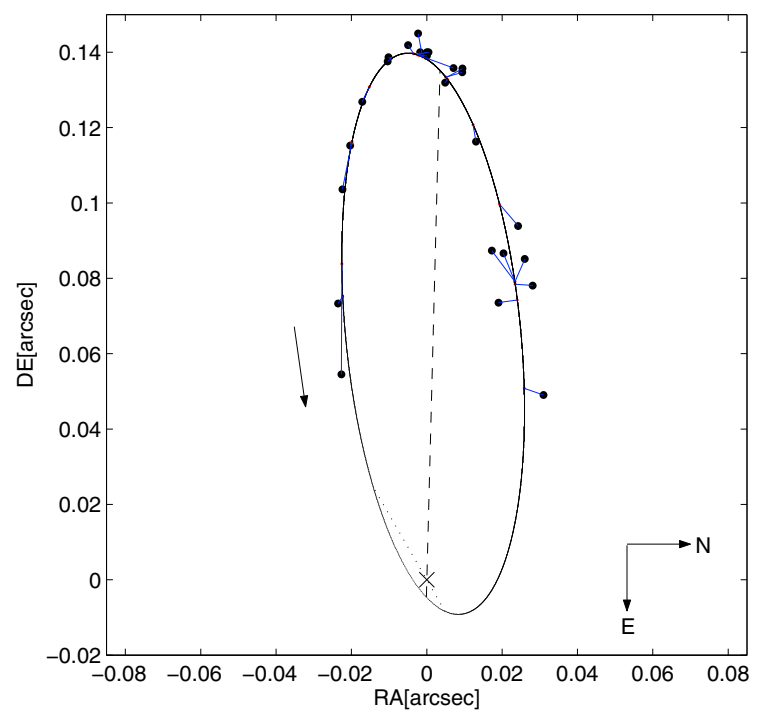

Fig. 4. Orbit of A-B pair on a plane of the sky. The dashed line represents the line of the apsides, while the dotted one stands for the line of the nodes. The cross indicates the position of the eclipsing binary.

(or parallax) to the system and compare it to the Hipparcos one. Therefore, the set of parameters to be computed is the following: $\mathrm{HJD}_{0}, P, p_{3}, T_{0}, i, a, \omega, \Omega, e_{3}, A$, where $a$ denotes the semimajor axis in arcseconds, while $A$ stands for the semiamplitude of LITE. We used the least-squares method and the simplex algorithm (see e.g., Kallrath \& Linnell 1987). One can also discuss the use of quadratic ephemeris because of some mass transfer between the components, but this was not used in our analysis due to insufficient coverage of the $\mathrm{O}-\mathrm{C}$ diagram with data points in longer time scales. The two minima times based on Hipparcos data were only roughly estimated and therefore their use for the analysis is questionable.

The analysis results in a set of parameters presented in Table 4, where we list all computed values and some of the derived quantities. The final fits are presented in Figs. 4 and 5. For plotting the $\mathrm{O}-\mathrm{C}$ diagram, the individual minima in different filters were averaged to one point for better clarity. Figure 6 shows the residuals after subtraction of the fit. As one can see, some long-term variation cannot be ruled out with the current data. This could be caused e.g., by a mass transfer between the components, but only future observations can confirm or rule out

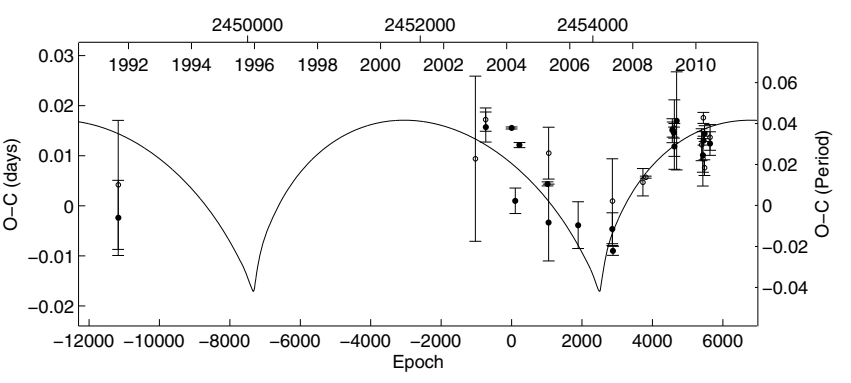

Fig. 5. O-C diagram of KR Com. The primary minima are plotted as dots, the secondary ones as open circles. It is obvious that the respective errors of the published minima times should be larger than presented.

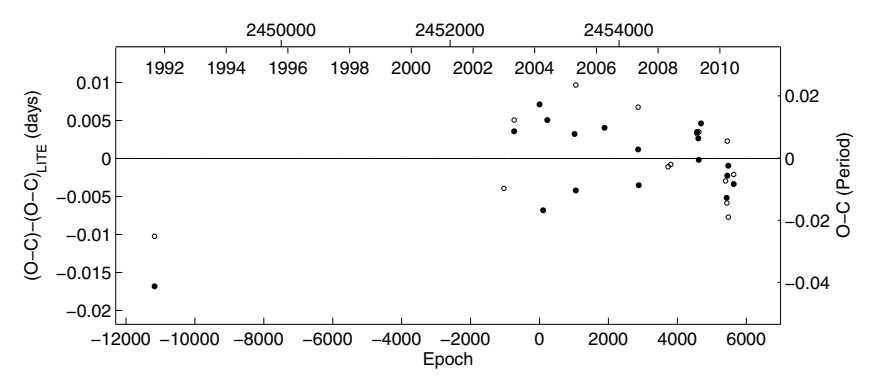

Fig. 6. O-C diagram of residuals of KR Com.

this possibility. Another potential explanation are magnetic cycles with a period of about 20 years (Applegate 1992), which cause not only a brightness variation, but also this period variation. A combination of both mass transfer and magnetic cycles is also possible.

The distance to the system was derived from the fit, resulting in $d=64.02 \pm 9.42 \mathrm{pc}$, closer than from the Hipparcos data. However, this value is only roughly estimated because it is derived from comparing the values of $A$ and $a$, but the detection of the LITE variation and deriving its amplitude is still problematic, and the $11 \mathrm{yr}$ period is not yet sufficiently covered by data. Due to this reason, and due to the relatively large difference between our derived parallax and the value from Hipparcos, we introduced Fig. 7. In this figure we fixed the semimajor axis of the visual orbit (this value is much better defined than the amplitude of LITE) and plotted the values of the parallax $\pi$ and mass of the third component $M_{3}$ with respect to the amplitude $A$ of LITE. As one can see, our resulting value of $A=0.0171$ days yields the values of $M_{3}$ and $\pi$ given in Table 4 . If we consider the range of potential values of $A$ according to its error, the values of $\pi$ are still far from the value derived by Hipparcos. Moreover, the mass of the third body with a decreasing value of parallax is growing rapidly. On the other hand, if we presume that the mass of the third component is lower, about e.g., $1.1 M_{\odot}$, then the amplitude of LITE is only about 0.013 days (which cannot reliably describe the fit in the $\mathrm{O}-\mathrm{C}$ diagram) and the parallax results in approximately 16.5 mas, which is even more distant than the Hipparcos value.

The other quantity, which can be compared with the previous results, is e.g., the mass of the distant component $M_{3}$. D'Angelo et al. (2006) presented a value about $1.19 M_{\odot}$, while our estimation is a bit higher, about $1.60 M_{\odot}$. This value has been derived from the assumption that the masses of the individual components are $M_{1}=1.42 M_{\odot}$ and $M_{2}=0.129 M_{\odot}$, which have been taken as constant input values. The resulting value of $M_{3}$ is surprising, especially if we take into consideration that the luminosities in all filters resulted in slightly lower values (B: $46 \%$, 


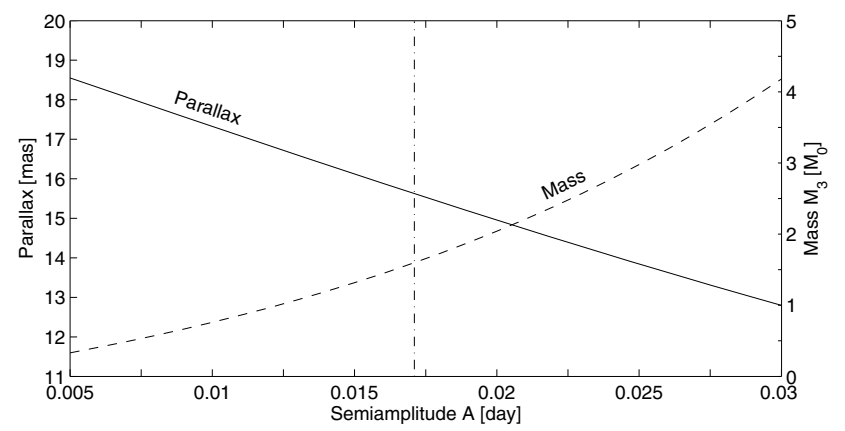

Fig. 7. Diagram of semiamplitude of LITE versus mass of the third body and parallax of the system with the fixed semimajor axis of the visual orbit. The dash-dotted line represents the value of $A$ from our final solution.

$V: 50 \%, R: 51 \%$ of $\left.L_{1}+L_{2}\right)$ than assumed by D'Angelo et al. (2006). If the mass of the third body is higher and the luminosity lower, this could indicate that this component is also a binary, or the star is underluminous than one would expect for a star of the main sequence.

\section{Discussion and conclusions}

The triple system KR Com revealed some of its basic physical properties for the first time. The light curve with shallow minima (due to a bright third component) shows that the system has a relatively low inclination of about only $52^{\circ}$. That we know the inclinations of both orbits is one aspect of KR Com system's uniqueness.

On one hand, a difference between the inclination of the close pair and the inclination of the orbit of the third distant component $\left(i_{3}=67.8^{\circ}\right)$ is still relatively small, therefore one could speculate about a common origin of all three components (see e.g., Zakirov 2008). On the other hand, a presence of two orbits which are not strictly coplanar is a necessary condition for a so-called Kozai cycles (Kozai 1962), which could play a role in the formation of this system. As mentioned by D'Angelo et al. (2006), the typical product of the Kozai mechanism is a close pair and a distant component on a non-coplanar orbit, which is the case here.

The other computable quantity in this triple system is its nodal period, or the precession of the orbits, which can change the inclination of the close eclipsing pair and therefore also the depths of the minima, see e.g., Mayer (2005). Regrettably, the nodal period here results in a value of about $100000 \mathrm{yr}$.

Today there are only 34 eclipsing binary systems known among the visual double stars that have known visual orbits. In all these systems one should expect a period variation of times of minima observed for these systems, but surprisingly, the LITE that agrees with the visual orbit has been detected in only seven systems (i Boo, VW Cep, $\zeta$ Phe, V819 Her, V772 Her, QS Aql, V505 Sgr). The system KR Com seems to be another example. The visual orbit with a period about $11 \mathrm{yr}$ is well-covered by interferometric data, and the combined solution of visual orbit and $\mathrm{O}-\mathrm{C}$ diagram of minima times is also dominated by the visual orbit. Therefore, the very first minima estimates based on Hipparcos data with their large errors were almost useless for the analysis. The other interesting fact is that the already published minima show much larger residuals than their published errors.
However, the combined fit brings new light into the system and confirms for example the $\omega$ and $\Omega$ values. Because the same fit to the interferometric data could be realized by changing both angles by $180^{\circ}$ (orientation of the orbit towards the observer), the LITE fit helps us to confirm the orientation of the orbit in the space without any need for spectroscopy. Nevertheless, the spectroscopic data and new times of minima would be also very profitable, especially in the upcoming periastron passage, which will occur in 2017.

Acknowledgements. This investigation was supported by the Research Programme MSM0021620860 of the Czech Ministry of Education. We thank P. Svoboda for providing us his photometric CCD observations. We also do thank the ASAS team for making all of the observations easily public available. An anonymous referee is also acknowledged for his or her helpful and critical suggestions, which greatly improved the paper. This research has made use of the SIMBAD and VIZIER databases, operated at CDS, Strasbourg, France and of NASA's Astrophysics Data System Bibliographic Services.

\section{References}

Abt, H. A. 1981, ApJS, 45, 437

Applegate, J. H. 1992, ApJ, 385, 621

Balega, I. I., Balega, Y. Y., Belkin, I. N., et al. 1994, A\&AS, 105, 503 Bessell, M. S. 1990, PASP, 102, 1181

D’Angelo, C., van Kerkwijk, M. H., \& Rucinski, S. M. 2006, AJ, 132, 650

Ducourant, C., Le Campion, J. F., Rapaport, M., et al. 2006, A\&A, 448, 1235

Fu, H.-H., Hartkopf, W. I., Mason, B. D., et al. 1997, AJ, 114, 1623

Irwin, J. B. 1959, AJ, 64, 149

Hall, D. S. 1989, Space Sci. Rev., 50, 219

van Hamme, W. 1993, AJ, 106, 2096

Harmanec, P. 1988, BAICz, 39, 329

Hartkopf, W. I., McAlister, H. A., \& Franz, O. G. 1992, AJ, 104, 810

Hartkopf, W. I., McAlister, H. A., Mason, B. D., et al. 1994, AJ, 108

Hartkopf, W. I., Mason, B. D., \& McAlister, H. A. 1996, AJ, 111, 370

Hartkopf, W. I., McAlister, H. A., \& Mason, B. D. 2001, AJ, 122, 3480

Horch, E. P., van Altena, W. F., Cyr, W. M., et al. 2008, AJ, 136, 312

Houdashelt, M. L., Bell, R. A., \& Sweigart, A. V. 2000, AJ, 119, 1448

Kallrath, J., \& Linnell, A. P. 1987, ApJ, 313, 346

Kazarovets, E. V., Samus, N. N., Durlevich, O. V., et al. 1999, IBVS, 4659, 1

Kozai, Y. 1962, AJ, 67, 591

Krajci, T. 2005, IBVS, 5592, 1

Kurucz, R. L. 1996, M.A.S.S., Model Atmospheres and Spectrum Synthesis, 108,2

Kwee, K. K., \& van Woerden, H. 1956, BAN, 12, 327

van Leeuwen, F. 2007, A\&A, 474, 653

Mason, B. D., Hartkopf, W. I., Gies, D. R., Henry, T. J., \& Helsel, J. W. 2009, AJ, 137, 3358

Mayer, P. 2005, Ap\&SS, 296, 113

McAlister, H. A., Hartkopf, W. I., Hendry, E. M., Campbell, B. G., \& Fekel, F. C. 1983, ApJS, 51, 309

McAlister, H. A., Hartkopf, W. I., Hutter, D. J., \& Franz, O. G. 1987, AJ, 93, 688

McAlister, H. A., Hartkopf, W. I., Sowell, J. R., Dombrowski, E. G., \& Franz, O. G. 1989, AJ, 97, 510

McAlister, H., Hartkopf, W. I., \& Franz, O. G. 1990, AJ, 99, 965

Nagai, K. 2006, VSOLB, 44, 1

Nagai, K. 2009, VSOLB, 48, 1

Perryman, M. A. C., \& ESA 1997, The HIPPARCOS and TYCHO catalogues, Astrometric and photometric star catalogues derived from the ESA Hipparcos Space Astrometry Mission (Noordwijk, Netherlands: ESA Publications Division), ESA SP Ser., 1200

Pojmanski, G. 2002, AcA, 52, 397

Press, W. H., Flannery, B. P., \& Teukolsky, S. A. 1986 (Cambridge: University Press)

Prša, A., \& Zwitter, T. 2005, ApJ, 628, 426

Rapaport, M., Le Campion, J.-F., Soubiran, C., et al. 2001, A\&A, 376, 325

Rucinski, S. M., Lu, W., Capobianco, C. C., et al. 2002, AJ, 124,

Selam, S. O. 2004, A\&A, 416, 1097

Tody, D. 1993, in IRAF in the Nineties in Astronomical Data Analysis Software and Systems II, ed. R. J. Hanisch, R. J. V. Brissenden, \& J. Barnes, ASP Conf. Ser., 52, 173

Wilson, R. E., \& Devinney, E. J. 1971, ApJ, 166, 605

Zakirov, M. M. 2008, Kinemat. Phys. Celest. Bodies, 24, 25

Zasche, P., \& Wolf, M. 2007, AN, 328, 928 